\title{
Geoinformation monitoring of crop productivity using intelligent analysis of vegetation indices
}

\author{
Kirill Tokarev ${ }^{1,2,}$, Yulia Orlova ${ }^{1}$, Vsevolod Kuzmin $^{2}$, and Tatyana Pleshenko ${ }^{2}$ \\ ${ }^{1}$ Volgograd State Technical University, 28, Lenina Avenue, Volgograd, 400005, Russia \\ ${ }^{2}$ Volgograd State Agricultural University, 26, University Avenue, Volgograd, 400002, Russia
}

\begin{abstract}
One of the most promising areas of development of the system for managing programmed agricultural production and increasing its productivity at present in Russia and abroad is the use of remote monitoring and sensing systems for subsequent interpretation of the data obtained by calculating and visualizing vegetation indices using intelligent systems. Using geomonitoring systems allows you to monitor the state of crops, make timely decisions on the implementation of appropriate agricultural measures. The development of the remote monitoring system, due to the increase in the number of satellites and the formation of available space survey data, suggests the possibility of creating an effective system for monitoring agricultural land and assessing crop yields.
\end{abstract}

\section{Introduction}

Assessment of the state of vegetation cover can be carried out on based on the spectral reflectivity of plants, which it is characterized by differences in the reflection of radiation of different wavelengths.

Knowledge of the relationship between the structure and state of vegetation and its reflective properties abilities allow you to use aerospace images for mapping and identification of vegetation types and their stress states. Spectral information is interpreted in the form of so-called "index" images. They are based on a combination of values brightness in certain channels that are informative for highlighting the subject under study of the object, and calculation of the "spectral index" of the object based on these values.

Each pixel of the image contains information about the index value, based on these indicators, it is possible to assess the state of the plant cover for certain characteristics in the user's area of interest regions. Spectral indices used for state estimation vegetation, received the generally accepted name of vegetation indices. Currently, about 160 vegetation options have been calculated indexes, among which there are groups that allow you to evaluate certain indicators of vegetation state.

*Corresponding author: tke.vgsha@mail.ru 


\section{Materials and methods}

The main features of the tasks to be solved for assessing the state of crops and soil cover using satellite monitoring and remote sensing data are:

- the components of the system "surface layer of the atmosphere - vegetation cover - soil cover" should be considered as a single complex, which will allow achieving sufficient accuracy and reliability of the assessment.

- the presence of two fundamentally different assessment modes: within the test site, where we have additional ground measurements; within the served region, where we can only have information coming from the remote monitoring system;

- remote monitoring systems should be equipped with multi-channel optical systems, which will allow you to choose the most effective data transmission channels for solving evaluation tasks.

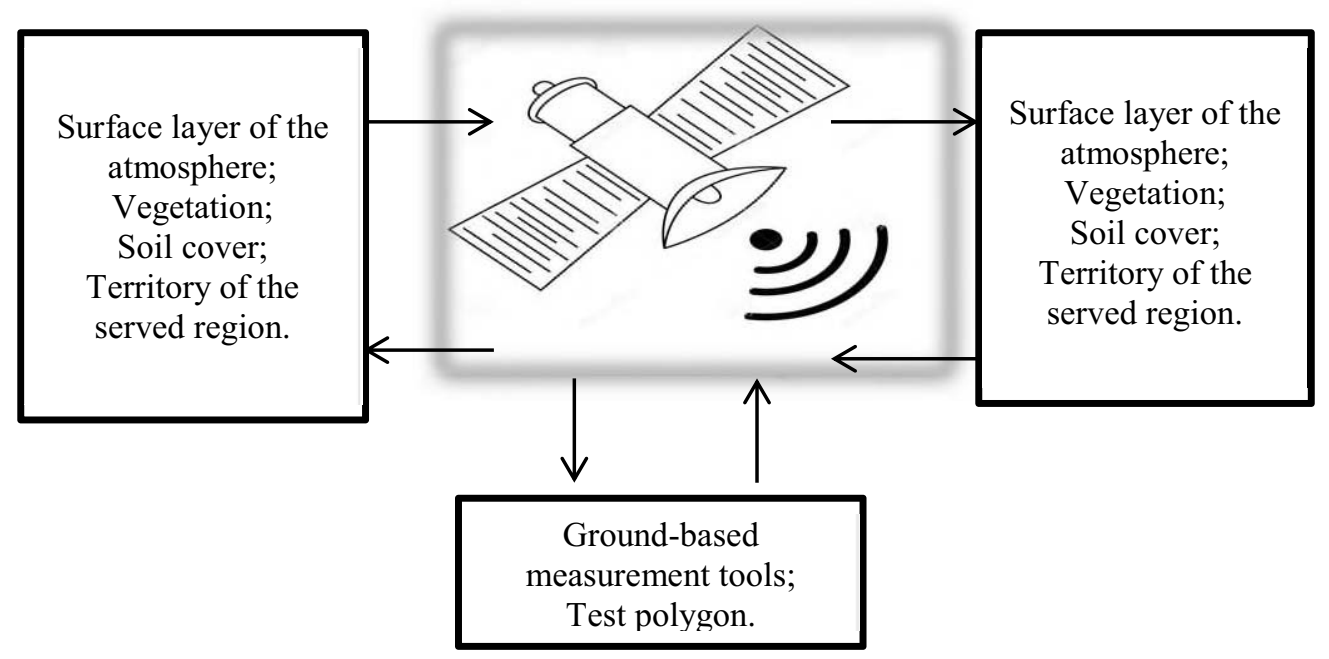

Fig. 1. Conceptual scheme for assessing the state of crops and soil cover.

Figure 2 shows a structural and algorithmic scheme for assessing the state of crops and soil cover using satellite monitoring data. The optimal scenario for assessing the state of crops and soil cover is the use of special procedures for processing a posteriori information obtained from remote monitoring tools and a priori information generated by dynamic mathematical models.

Broadband group greenness indexes are used for determining areas covered by vegetation, monitoring vegetation cover, determining crop yields, and mapping. The calculation can be performed using images of any resolution that have spectral channels in the red (0.60 - 0.75 microns) and near-infrared (0.75-1.3 microns) zones. The narrow-band group of greenness indices is used to assess the state of vegetation and reflects its total number. A distinctive feature is the calculation of coefficient values in the cross-section of the spectrum from 0.69 to 0.75 microns. The advantage is to detect the smallest changes in the state of vegetation cover. The calculation is performed only when using hyperspectral images. Light efficiency - indicators that allow you to evaluate the efficiency of light use by plants for photosynthesis processes. Plant growth and productivity are evaluated using indicators of this group. Nitrogen canopy is an indicator that determines the nitrogen content in plants. Fast-growing vegetation is characterized by a high nitrogen content, while at low concentrations, the leaves turn pale green, become smaller, and the branching of shoots decreases. The average infrared range is used to calculate the relative nitrogen 
content in vegetation. Dry or aging carbon indexes are designed to account for the total amount of "dry" carbon in the form of lignin and cellulose. An increase in these indicators may reflect the process of "aging" and death of plants. Average infrared radiation is used to calculate the relative nitrogen content in vegetation. It is widely used in assessing fire safety on the territory. Leaf pigments are indicators that reflect the content of carotenoid and anthocyanin pigments, which are observed in significant quantities in depressive vegetation. In agriculture, indexes of this group are used for monitoring vegetation conditions, determining field stress zones, and predicting data in narrow spectral zones of the visible range. Moisture content in the canopy - indicators that reflect the humidity of plants. The near and mid-infrared ranges are used to calculate the indices. Indexes are widely used in assessing fire safety in the territory together with indexes of dry or aging carbon group. Vegetation values are calculated based on the values of the two most stable sections of the spectral reflectivity curve of plants. The red zone of the spectrum $(0.62-0.75$ microns) is characterized by the maximum values of absorption of solar radiation by chlorophyll, and the near - infrared zone of the spectrum (0.75-1.3 microns) accounts for the maximum reflection of energy by the cell structure of the leaf [1-5, 9].

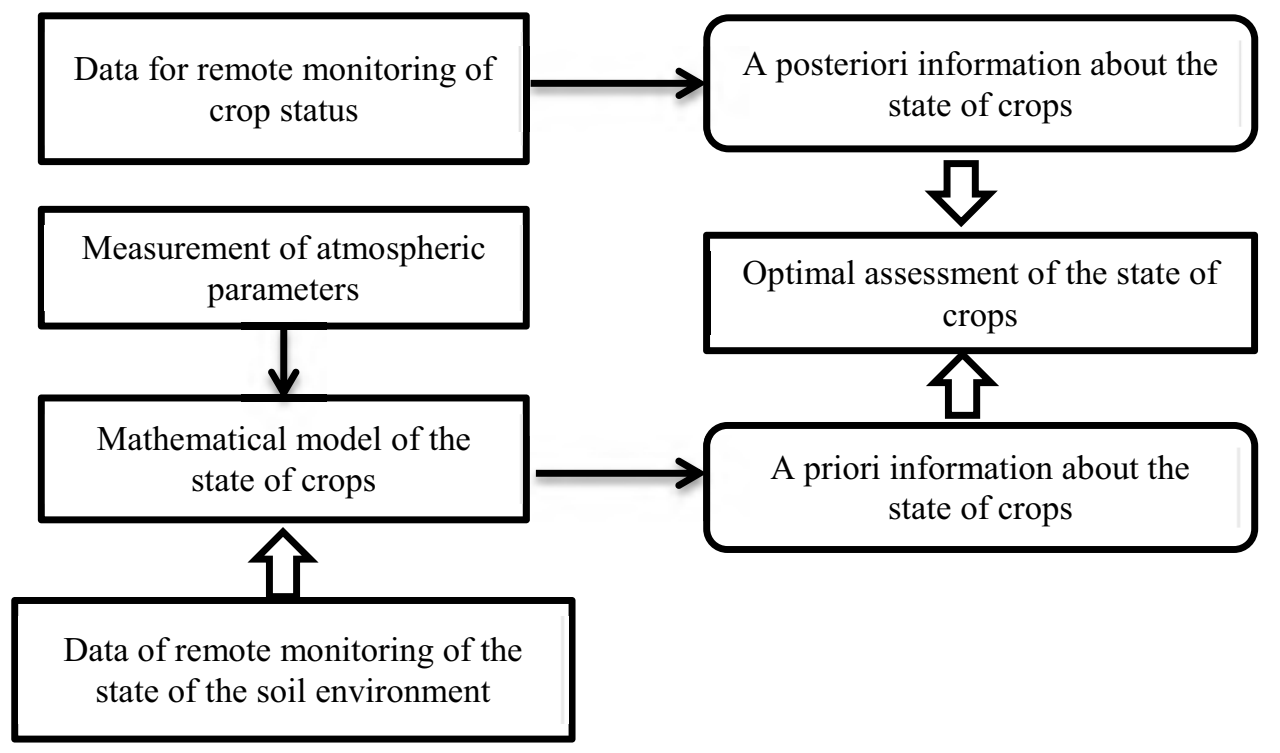

Fig. 2. Structural and algorithmic scheme for assessing the state of crops.

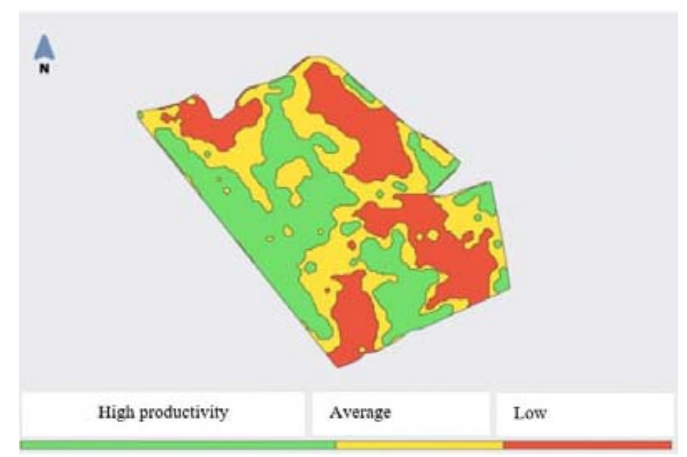

Fig. 3. Productivity zones of the studied field.

The most well - known plant index is the normalized difference plant index-the 
normalized difference plant index is the most common in use. This is a simple graphical indicator that is used to assess the quantitative and qualitative characteristics of vegetation cover. Due to the presence of chlorophyll in the cells of green plants, they are able to absorb solar radiation in the spectral region of photosynthetically active radiation (from 0.4 to 0.7 microns), which is used as an energy source during photosynthesis. Plant cells are also able to reflect solar radiation in the near-infrared region of the spectrum (from 0.7 to 1.1 microns). That is, high photosynthetic activity (usually associated with dense vegetation) leads to less reflection in the red region of the spectrum and more in the infrared (figure 3 ). The ratio of these indicators to each other allows you to clearly separate and analyze vegetation from other natural objects. Using a normalized difference between the minimum and maximum reflections instead of a simple ratio increases the accuracy of measurements and reduces the impact of phenomena such as differences in image illumination, cloud cover, haze and radiation absorption by the atmosphere. The NDVI value changes throughout the season depending on the stage of plant development. The beginning of the growing season is characterized by an increase in the index value, which, having reached its peak at the time of flowering, stops. It is worth noting that the rate of development of biomass varies depending on weather conditions, soil fertility and crop cultivation technologies. Therefore, the average NDVI value for the field will allow you to compare the state of cultivated crops during the growing season: in some fields, crops develop faster in others-slower.

\section{Results}

To implement more accurate crop yield forecasting, it is necessary to take into account the NDVI value at the time of its peak maturation, that is, the maximum value. Thus, with intensive cultivation of winter wheat, the NDVI value during the peak reaches $0.80-0.88$, usually at the beginning of the earing phase. Knowing the potential yield of a variety, you can make a forecast at which value of the NDVI index the yield will be maximum for this variety. The NDVI value of $0.60-0.65$ during the earing phase of winter wheat indicates that the yield will be $25-30 \%$ lower than the maximum. This is due to the ratio between NDVI and green plant biomass, the percentage of which is an indicator of yield.

Thus, to predict the yield, it is necessary to have information about the maximum potential yield of the cultivated crop variety, as well as to take into account the value of the NDVI index in the earing phase for cereals or in the phase of maximum leaf development for all other crops. NDVI can be calculated based on any high -, medium-or low-resolution images with spectral channels in the red (0.55-0.75 microns) and infrared (0.75-1.0 microns) ranges. It is worth noting that it can be calculated using software that has a built-in index calculation module. The NDVI calculation algorithm is built into almost all common software packages related to remote sensing data processing (ArcView Image Analysis, ERDAS Imagine, ENVI, Ermapper, Scanex MODIS Processor, ScanView, etc.) (figure 4).

To use NDVI effectively when calculating productivity and other quantitative indicators, it is necessary to compare the obtained NDVI values with pre-collected data from test sites, which must take into account seasonal environmental and climatic indicators of both the image itself and the test sites at the time of data collection. Also, one of the disadvantages of this index is the ability to use survey data only during the growing season of the studied plants. This is due to the fact that NDVI is tied to the amount of photosynthetic biomass and is ineffective in images obtained during the season of weakened or non-vegetating vegetation during this period. You should take into account the fact that the values of the received vegetation indices depend on the sensor characteristics (spatial and spectral resolution), illumination, and shooting conditions, so they cannot 
provide absolute quantitative indicators of the studied vegetation characteristics. To accurately interpret them, it is necessary to use data from field measurements [6-8,10-12].

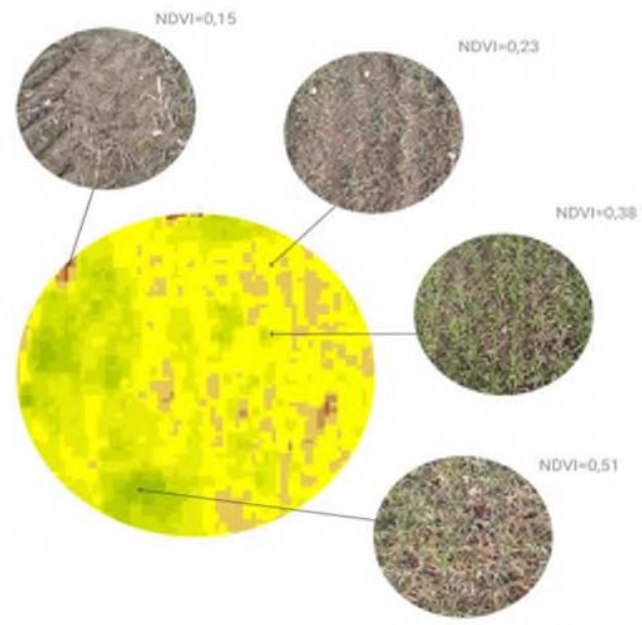

Fig. 4. NDVI visualization results and their correlation with the actual state of crops.

\section{Conclusion}

Geomonitoring systems and remote sensing images used to monitor the state of crops in large agricultural fields, which can be processed to track the state of crops in specific fields. Correct interpretation of satellite image data allows the user to make decisions about "spot" deposits of fertilizers, insecticides or herbicides, irrigation and other activities. A distinctive feature of satellite images is a high degree of visibility, covering large areas of the surface in a single image. depending on the type of equipment and films used, photography can be performed in the entire visible range of the electromagnetic spectrum, in its individual zones, as well as in the near-infrared range. Satellite images used for crop forecasting they must meet certain requirements for their further operation processing and analysis. Basic parameters of satellite images, determining their quality and usability, these are:

- spatial resolution that determines the maximum possible accuracy of displaying acreage. High resolution data satellite imagery allows you to get more accurate geometric data characteristics as a result of their analysis;

- spectral resolution. The requirements to the spectral resolution due to the use of channels in red (0.6-0.7 microns) and near infrared (0.75-0.90 microns) spectral ranges. In these ranges there is a calculation of the normalized relative index of vegetation - NDVI, which allows characterize the state of vegetation in agricultural fields;

- temporary resolution, which characterizes the frequency of conducting shooting. For agricultural tasks, it is recommended to take a survey with frequency of 7-9 days throughout the year.

There are many satellites whose survey equipment meets the requirements listed above. To implement monitoring satellite data can be used on agricultural land LANDSAT, TERRA, SPOT, IRS-1D, "Comet", "QuickBird". In the nearest in the future, it is planned to implement operational data acquisition wide-area satellite systems, which will allow for continuous monitoring of monitoring of large territories. As an example, you can give Deimos-1 (Spain), DMC-2 (UK), Resourcesat-2 satellites (India). To perform a temporary analysis of state changes archived data can be used for agricultural land satellites SPOT 2, 
SPOT 4, Landsat 4, Landsat 5, Landsat 7. Data archives contain information for more than 20 years.

The research was conducted as a part of the grant of the President of the Russian Federation MK592.2020.11.

\section{References}

1. R. E. Crippen, Calculating the Vegetation Index Faster, Remote Sensing of Environment, 34, 71-73 (1990)

2. B. Datt A New Reflectance Index for Remote Sensing of Chlorophyll Content in Higher Plants: Tests Using Eucalyptus Leaves, Journal of Plant Physiology, 154, 3036 (1999)

3. J. A. Gamon, L. Serrano and J. S. Surfus The Photochemical Reflectance Index: An Optical Indicator of Photosynthetic Radiation Use Efficiency Across Species, Functional Types and Nutrient Levels, Ecology, 112(4), 492-501 (1997)

4. I. M. Mikhailenko Assessment of crop and soil state using satellite remote sensing data International, Journal of Information Technology \& Operations Management, 1(5), 41-52 (2013)

5. V. Veklenko, et al. Designing a database of models for optimal planning of agricultural production, Bulletin of the Kursk state agricultural Academy, 1, 33-36 (2013)

6. M. Deepak, et al., Leaf Canopy Layers Affect Spectral Reflectance in Silver Birch, Remote Sens, (11) 2884 (2019)

7. S. Lysenko Forecasting crop yields based on satellite monitoring of carbon dynamics in terrestrial ecosystems, Exploring the Earth from space, 4, 48-59 (2019)

8. A. Aksenov Analysis of intelligent decision support systems in agriculture, Electrotechnology and electrical equipment in agriculture, 3(36), 46-51 (2019)

9. D.A. Sims, J.A. Gamon Relationships Between Leaf Pigment Content and Spectral Reflectance Across a Wide Range of Species, Leaf Structures and Developmental Stages, Remote Sensing of Environment, 61, 337-354 (2002)

10. K. E. Tokarev, Yu. A. Orlova, A. F. Rogachev, V. A. Kuzmin and Yu. M. Tokareva Crops reclamation management based on hybrid neuro-fuzzy systems, IOP Conf. Series: Earth and Environmental Science, 421, 042015 (2020)

11. K. E. Tokarev, et al., The intelligent analysis system and remote sensing images segmentation engineering by using methods of advanced machine learning and neural network modelling, IOP Conf. Series: Materials Science and Engineering, 734, 012124 (2020)

12. E. V. Melikhova and A. F. Rogachev Computer simulation and optimization of parameters of configuration of the contour of moistening under drip irrigation of agricultures, Studies in Computational Intelligence, 826, 1193-1201 (2019) 